\title{
Investigation of Early Death-Induced Changes in Rat Brain by Solid Phase Microextraction via Untargeted High Resolution Mass Spectrometry: In Vivo versus Postmortem Comparative Study
}

\author{
Sofia Lendor, ${ }^{1}$ Mariola Olkowicz, ${ }^{1, \div}$ Ezel Boyaci, ${ }^{1, \dagger}$ Miao Yu, ${ }^{1,}, *$ Mustansir Diwan, ${ }^{2,+}$ Clement Hamani, ${ }^{2,+}$ Michael Palmer, ${ }^{1}$ \\ Nathaly Reyes-Garcés, ${ }^{1, \ddagger}$ German Augusto Gómez-Ríos, ${ }^{1, \neq}$ and Janusz Pawliszyn ${ }^{1, *}$ \\ ${ }^{1}$ Department of Chemistry, University of Waterloo, Waterloo, Ontario, Canada N2L 3G1 \\ 2 Neuroimaging Research Section, Centre for Addiction and Mental Health, 250 College Street, M5T 1R8, Toronto, ON, Canada \\ Current addresses: \\ $\div$ Jagiellonian Centre for Experimental Therapeutics (JCET), 30-348 Kraków, Poland \\ † Department of Chemistry, Middle East Technical University, Ankara, 06800, Turkey \\ $¥$ Department of Environmental Medicine and Public Health, Icahn School of Medicine at Mount Sinai, New York, NY, USA \\ + Harquail Centre for Neuromodulation, Sunnybrook Research Institute, Sunnybrook Health Sciences Centre, 2075, Bayview Avenue, \\ Toronto, ON, Canada, M4N 3M5 \\ ‡ Restek Corporation, Bellefonte, PA, USA, 16823 \\ *Corresponding author; Email: janusz@uwaterloo.ca; Tel:519-888-4567; Fax: 519-746-0435
}

\section{Table of Contents}

LC-MS methods details

Multivariate statistical analysis plots

Data transformation and distribution

List of metabolites showing statistically significant differences among in vivo and postmortem

samplings in the hippocampus at different time points.

Boxplots for the discussed metabolites.

Confirmation of metabolites' identities by MS/MS - comparison with real standards ...

Confirmation of metabolites' identities by MS/MS - comparison with databases

MS/MS fragments related to metabolites which could not be confirmed by comparison with real standards or database search.

References 


\section{LC-MS methods details}

Table S1: MS acquisition parameters and LC method details for analysis of metabolites and lipids in rat brain SPME extracts.

\begin{tabular}{|c|c|c|}
\hline \multicolumn{3}{|c|}{ High Performance Liquid Chromatography } \\
\hline & Reversed-phase & HILIC \\
\hline Instrument & Thermo Accela 1250 & Thermo Vanquish Flex \\
\hline Column & $\begin{array}{l}\text { Supelco Discovery HS F5, } 3 \mu \mathrm{m}, 100 \mathrm{x} \\
2.1 \mathrm{~mm}, 20 \times 2.1 \mathrm{~mm} \text { guard column }\end{array}$ & $\begin{array}{c}\text { Merck SeQuant ZIC-HILIC, } 3.5 \mu \mathrm{m}, 100 \times \\
2.1 \mathrm{~mm} \text { with } 20 \times 2.1 \mathrm{~mm} \text { guard column }\end{array}$ \\
\hline Mobile phase A & \multicolumn{2}{|c|}{$\mathrm{H}_{2} \mathrm{O}+0.1 \% \mathrm{FA}(+1 \mathrm{mM} \mathrm{AA})$} \\
\hline Mobile phase B & \multicolumn{2}{|c|}{$\mathrm{ACN}+0.1 \% \mathrm{FA}(+1 \mathrm{mM} \mathrm{AA})$} \\
\hline Flow rate $[\mu \mathrm{L} / \mathrm{min}]$ & 300 & 350 \\
\hline Column temperature $\left[{ }^{\circ} \mathrm{C}\right]$ & 25 & 40 \\
\hline Samples temperature $\left[{ }^{\circ} \mathrm{C}\right]$ & \multicolumn{2}{|c|}{5} \\
\hline Injection volume $[\mu \mathrm{L}]$ & \multicolumn{2}{|c|}{10} \\
\hline Desorption solvent & methanol/water $1: 1$ & acetonitrile/water $4: 1$ \\
\hline \multirow[t]{2}{*}{ Gradient [\%B] } & $\begin{array}{c}0 \text { min-0\%; } 3 \text { min-0\%; } 25 \text { min- } 90 \% ; 34 \text { min- } \\
\text { 90\%; } 35 \text { min-0\%; } 40 \text { min-0\% }\end{array}$ & $\begin{array}{c}0 \min -90 \% ; 2 \min -90 \% ; 22 \min -50 \% ; 27 \\
\min -50 \% ; 27.5 \min -90 \% ; 35 \min -90 \%\end{array}$ \\
\hline & \multicolumn{2}{|l|}{$\begin{array}{l}\text { Thermo Exactive Mass Spectrometer } \\
\text { (heated electrospray ionization source) }\end{array}$} \\
\hline Spray voltage $[\mathrm{kV}]$ & $4.0(-2.9)$ & $3.5(-3.2)$ \\
\hline Sheath gas & 55 & 60 \\
\hline Auxiliary gas & 30 & 20 \\
\hline Sweep gas & 5 & 2 \\
\hline Capillary temperature $\left[{ }^{\circ} \mathrm{C}\right]$ & $300(300)$ & $275(250)$ \\
\hline Vaporizer temperature $\left[{ }^{\circ} \mathrm{C}\right]$ & 300 & 300 \\
\hline Mass range $[\mathrm{m} / \mathrm{z}]$ & $100-1000$ & $75-1000$ \\
\hline Acquisition mode & \multicolumn{2}{|c|}{ Full Scan } \\
\hline Max. inject time [ms] & \multicolumn{2}{|c|}{100} \\
\hline AGC target & \multicolumn{2}{|c|}{ Balanced: $1 \mathrm{e} 6$} \\
\hline Resolution & \multicolumn{2}{|c|}{ High: 50,000@ @ 2Hz } \\
\hline Lock Mass & \multicolumn{2}{|c|}{ m/z 391.2843 (positive); m/z 255.2329 (negative) } \\
\hline
\end{tabular}

Negative ionization mode parameters in brackets; FA- formic acid; AA- acetic acid

Table S2: MS/MS acquisition parameters for confirmation of identities of significant metabolites and lipids found in rat brain extracts.

\begin{tabular}{cc} 
& $\begin{array}{c}\text { Thermo Q-Exactive Mass Spectrometer } \\
\text { (heated electrospray ionization source) }\end{array}$ \\
\hline Spray voltage [kV] & $3.5(-2.8)$ \\
Sheath gas & 35 \\
Auxiliary gas & 5 \\
Sweep gas & 0 \\
Capillary temperature $\left[{ }^{\circ} \mathrm{C}\right]$ & $300(300)$ \\
Vaporizer temperature [ $\left.{ }^{\circ} \mathrm{C}\right]$ & 300 \\
S-Lens RF level [V] & 60 \\
Acquisition mode & Parallel Reaction Monitoring \\
Max. inject time [ms] & $100(50)$ \\
AGC target & $1 \mathrm{e} 6$ \\
Resolution & 35,000 \\
Lock Mass & 20 and 40 \\
Normalized Collision Energy [V] & $\mathrm{m} / \mathrm{z} 391.28429$ (positive); m/z 255.23295 (negative)
\end{tabular}

Negative ionization mode parameters in brackets 


\section{Multivariate statistical analysis plots}
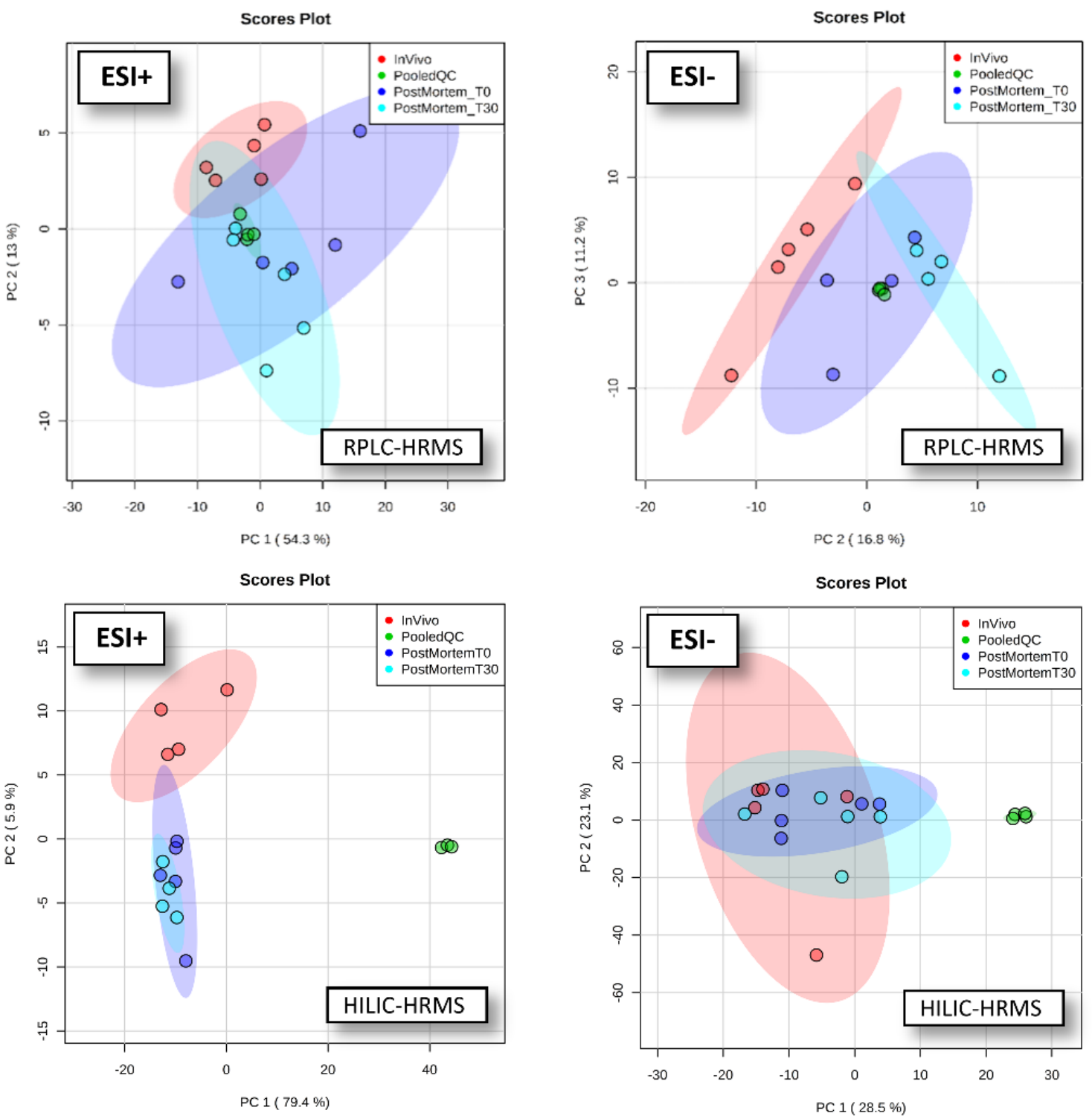

Figure S2. Principal component analysis (PCA) scores plots for analyses via RPLC-HRMS (top) and HILIC-HRMS (bottom) in positive and negative ionization modes (left and right, respectively). The plots display the first and second component and the group of pooled QC samples is included to prove stability and reliability of the instrumental analysis. 
A. In vivo vs. Post mortem To
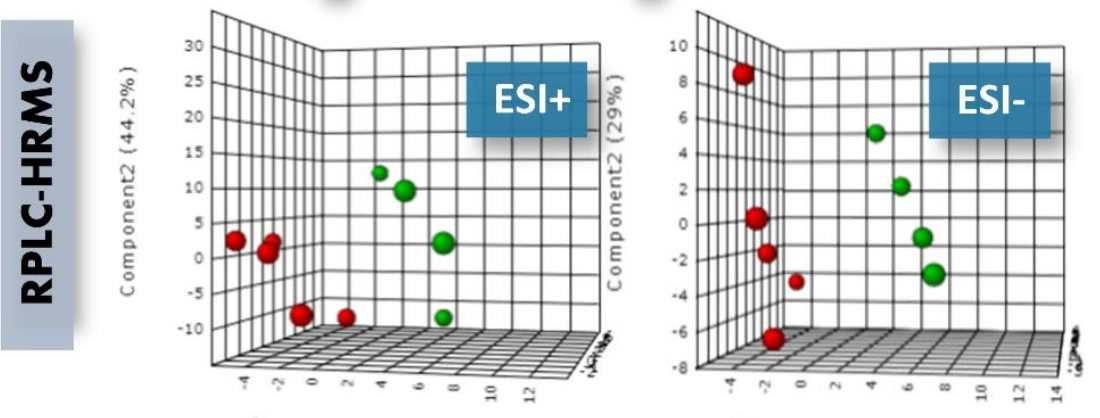

Component1 $(22.4 \%)$

Component1 (18.5\%)
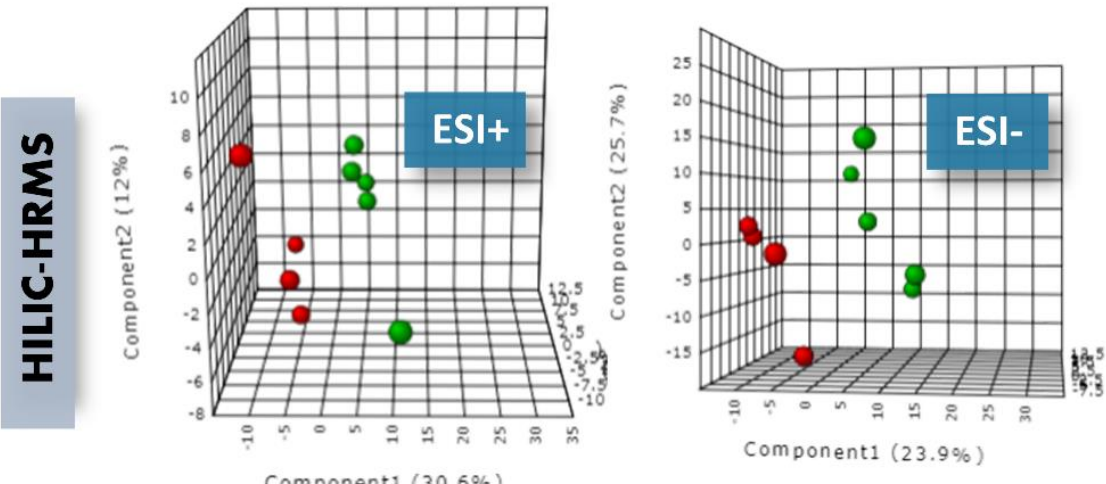

Component1 (30.6\%)

\section{B. Post mortem To vs. OPost mortem T30}
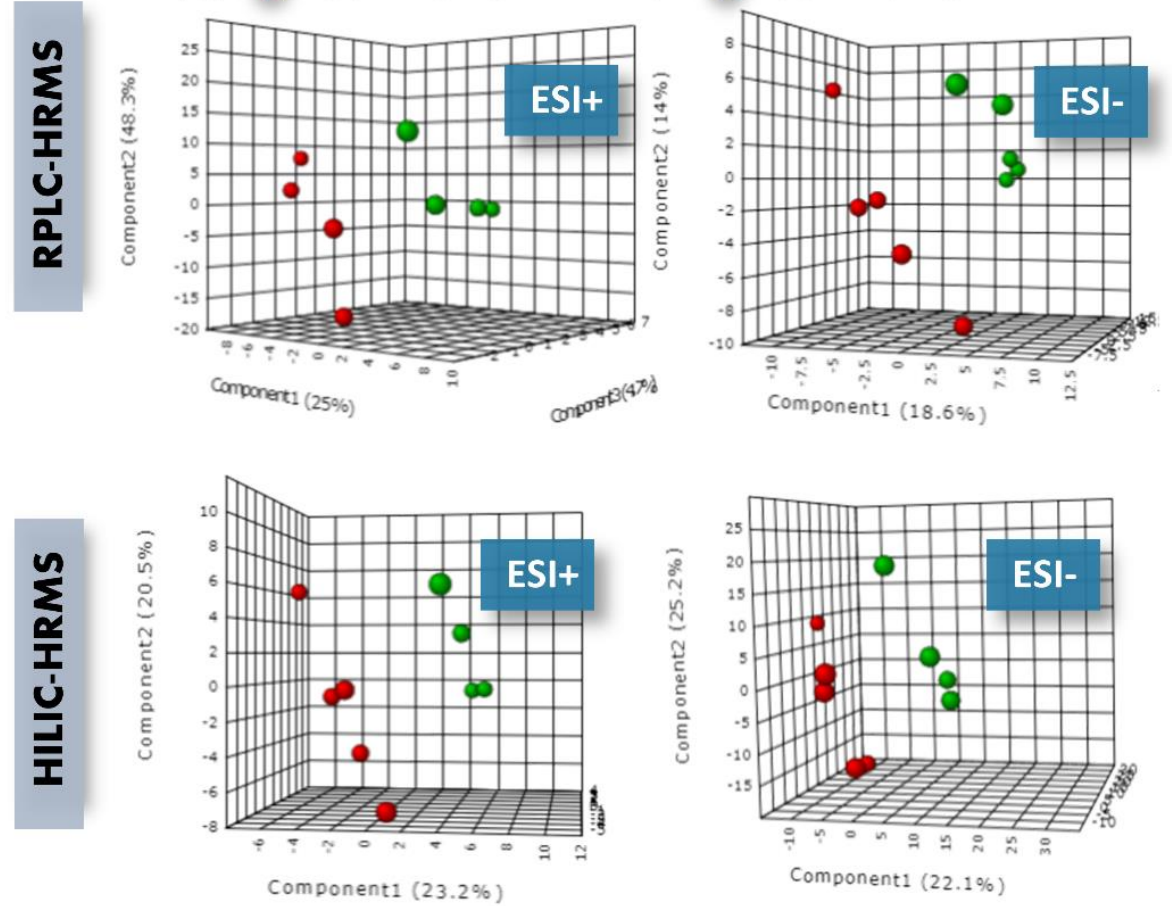

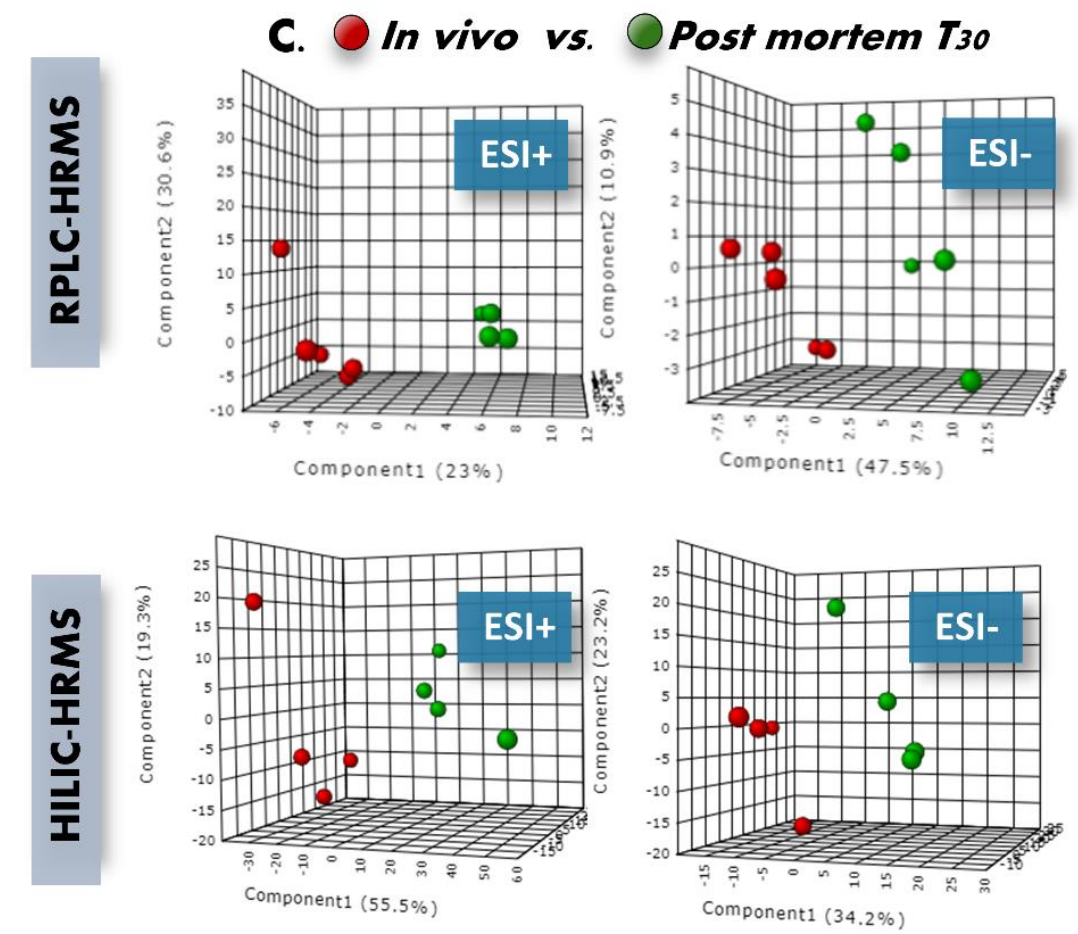

Figure S3. Two-group PLSDA for all combinations between the sampling time points: A. in vivo and postmortem T0; B. postmortem T0 and T+30; C. in vivo and postmortem $\mathrm{T}+30$. Amongst all models where clear separation is observed, only the comparisons between in vivo and postmortem at $\mathrm{T}+30$ passed validation using the LOOCV method. This implies that progressing changes in metabolomic profile become more significant and accurately predicted by PLSDA model as the PMI progresses.

\section{Data transformation and distribution}

After peak extraction, filling, grouping, alignment, and filling performed by the XCMS package, the output data table consists of features in rows and samples (here, 3 sampling timepoints, 5 animals in each) in columns; each cell contains peak area count, which is the initial analytical response. This response is subsequently transformed via logarithm transformation and auto scaling performed using MetaboAnalyst platform. The purpose of log transformation is to transform the response into a form that makes it comparable between different features. In LCMS-based metabolomic profiling of tissue extracts it is common that metabolites are present at drastically different concentration levels, often differing by several orders of magnitude. ${ }^{1}$ Since this scaling strategy is reported to not fully correct for the differences in magnitude of signals from different metabolites, auto scaling was also employed. Despite this strategy being recommended, it is not entirely clear how these combined data operations affect each other in the context of metabolomic data. ${ }^{2}$

Log transformation and auto scaling are performed according to equations 1 and 2 , respectively. ${ }^{2}$

$$
\begin{aligned}
& \tilde{x}_{i j}={ }^{10} \log \left(x_{i j}\right) \\
& \hat{x}_{i j}=\tilde{x}_{i j}-\overline{\tilde{x}}_{i} \\
& \tilde{x}_{i j}=\frac{x_{i j}-\bar{x}_{i}}{s_{i}}
\end{aligned}
$$

Where: $x_{i j}$ - response; $\bar{x}$-mean; $\hat{x}$ and $\tilde{x}$ - response after data pre-treatment steps; $s_{i}$-standard deviation. 
Ultimately, the data transformation creates a new response, which is subjected to subsequent statistical tests. The outlined data pre-treatment operations also transform the distribution of variables into one resembling normal distribution. The evidence that transformed data have distribution suitable for t-testing is provided as a visualization in the MetaboAnalyst platform. ${ }^{3}$
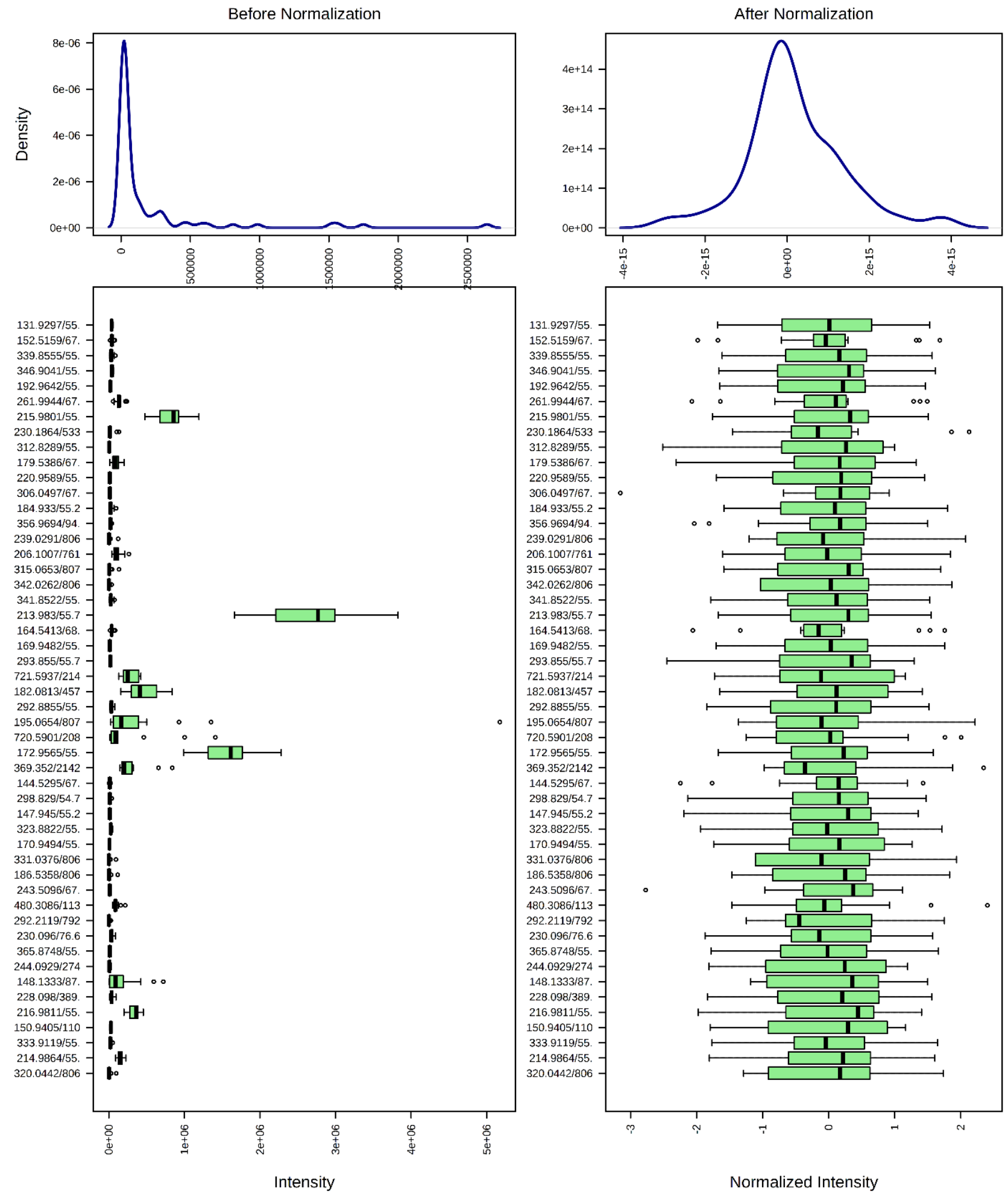

Figure S4. Data distribution (shown by feature) before (left) and after (right) pre-treatment operations including log transformation and auto scaling. Visualization was obtained by MetaboAnalyst platform via kernel density estimation. After transformation the data resembles normal distribution, suitable for t-testing. 
Table S3. List of metabolites showing statistically significant differences among in vivo and postmortem samplings in the hippocampus at different time points.

\begin{tabular}{|c|c|c|c|c|c|c|c|c|c|c|c|c|c|}
\hline \multirow{2}{*}{ Compound } & \multirow{2}{*}{$\begin{array}{c}\mathrm{Rt} \\
(\mathrm{min})\end{array}$} & \multirow{2}{*}{ LC method } & \multirow{2}{*}{$\mathrm{m} / \mathrm{z}$} & \multirow{2}{*}{ Adduct } & \multirow{2}{*}{$\begin{array}{c}\text { Mass error } \\
{[\mathrm{ppm}]}\end{array}$} & \multirow{2}{*}{ Level $\uparrow$} & \multicolumn{2}{|c|}{ IV vs. PM To } & \multicolumn{2}{|c|}{ PM To vs. $T_{30}$} & \multicolumn{2}{|c|}{ IV vs. PM T $T_{30}$} & \multirow{2}{*}{ Pathway/Function } \\
\hline & & & & & & & FC & $p$-value & FC & $p$-value & FC & $p$-value & \\
\hline \multirow{3}{*}{ 15-deoxy-delta-12,14-Prostaglandin J2 } & 15.2 & RPLC & 315.1968 & {$[\mathrm{M}-\mathrm{H}]^{-}$} & 1 & \multirow{3}{*}{3} & $\downarrow 2.45$ & 0.090 & $\downarrow 1.72$ & 0.023 & $\downarrow 4.23$ & 0.038 & \multirow{3}{*}{$\begin{array}{l}\text { Arachidonic acid metabolism/anti- } \\
\text { inflammatory prostaglandin }\end{array}$} \\
\hline & 0.84 & HILIC & 317.2115 & {$[\mathrm{M}+\mathrm{H}-\mathrm{H} 2 \mathrm{O}]+$} & 0 & & $\downarrow 1.68$ & 0.073 & $\downarrow 2.10$ & 0.085 & $\downarrow 3.54$ & 0.019 & \\
\hline & 0.81 & HILIC & 315.1966 & {$[\mathrm{M}-\mathrm{H}]^{-}$} & 0 & & $\downarrow 2.18$ & 0.031 & $\downarrow 1.35$ & 0.17 & $\downarrow 2.93$ & 0.021 & \\
\hline Prostaglandin & 15.3 & RPLC & 335.2218 & {$[\mathrm{M}+\mathrm{H}]^{+}$} & 0 & 1 & $\downarrow 3.03$ & 0.043 & $\downarrow 1.54$ & 0.11 & $\downarrow 4.67$ & 0.021 & Arachidonic acid metabolism \\
\hline Prostaglandin & 0.8 & HILIC & 333.2071 & {$[\mathrm{M}-\mathrm{H}]^{-}$} & 0 & \multirow[b]{2}{*}{1} & $\downarrow 2.61$ & 0.0071 & $\downarrow 1.16$ & 0.51 & $\downarrow 3.03$ & 0.0089 & \multirow[b]{2}{*}{ Arachidonic acid metabolism } \\
\hline $\begin{array}{l}\text { (cyclopentenone prostaglandin or } \\
\text { cyclopentenone isoprostane) }\end{array}$ & 0.8 & HILIC & 351.2175 & {$[\mathrm{M}-\mathrm{H} 2 \mathrm{O}-\mathrm{H}]^{-}$} & 0 & & $\downarrow 3.39$ & 0.011 & $\downarrow 1.42$ & 0.27 & $\downarrow 4.81$ & 0.013 & \\
\hline Prostaglandin & 0.8 & HILIC & 353.2333 & {$[\mathrm{M}-\mathrm{H}]-$} & 0 & 1 & $\downarrow 1.71$ & 0.033 & $\downarrow 1.08$ & 0.79 & $\downarrow 1.85$ & 0.0032 & Arachidonic acid metabolism \\
\hline FA 17:4 & 18.3 & RPLC & 263.2006 & {$[\mathrm{M}+\mathrm{H}-\mathrm{H} 2 \mathrm{O}]^{+}$} & 1 & 1 & $\downarrow 1.78$ & 0.095 & $\downarrow 1.44$ & 0.46 & $\downarrow 2.56$ & 0.013 & Arachidonic acid metabolism \\
\hline GlcCer(d40:1)/GalCer(d40:1) & 26.1 & RPLC & 784.6661 & {$[\mathrm{M}+\mathrm{H}]^{+}$} & 0 & 1 & 个 1.18 & 0.83 & 个 1.48 & 0.39 & 个 1.77 & 0.035 & Cell signal transduction \\
\hline GlcCer(d42:1)/GalCer(d42:1) & 26.8 & RPLC & 812.6974 & {$[\mathrm{M}+\mathrm{H}]^{+}$} & 0 & 1 & 个 1.35 & 0.28 & 个 1.40 & 0.045 & $\uparrow 1.86$ & 0.035 & Cell signal transduction \\
\hline $\begin{array}{l}\text { 5alpha-Androst-16-en-3-one or } \\
\text { 5alpha-Androst-3-en-17-one }\end{array}$ & 15.2 & RPLC & 271.2069 & {$[\mathrm{M}-\mathrm{H}]^{-}$} & 1 & 1 & $\downarrow 2.54$ & 0.089 & $\downarrow 1.76$ & 0.031 & $\downarrow 4.47$ & 0.039 & Cell signal transduction \\
\hline Adenosine monophosphate & 1.3 & RPLC & 348.0704 & {$[\mathrm{M}+\mathrm{H}]^{+}$} & 0 & 1 & $\downarrow 2.15$ & 0.022 & $\downarrow 1.86$ & 0.052 & $\downarrow 4.00$ & 0.0032 & Energy metabolism \\
\hline \multirow{2}{*}{ Creatine } & 2.3 & RPLC & 132.0769 & {$[\mathrm{M}+\mathrm{H}]^{+}$} & 1 & \multirow[b]{2}{*}{5} & 个 3.55 & 0.036 & 个 1.41 & 0.25 & 个 5.02 & 0.0087 & \multirow{2}{*}{ Energy metabolism } \\
\hline & 2.17 & RPLC & 130.0613 & {$[\mathrm{M}-\mathrm{H}]-$} & 6 & & $\uparrow 3.47$ & 0.076 & $\uparrow 1.48$ & 0.33 & 个 5.14 & 0.0015 & \\
\hline Phytanic Acid & 2.7 & HILIC & 330.3363 & {$[\mathrm{M}+\mathrm{NH} 4]^{+}$} & 1 & 1 & 个 1.17 & 0.74 & $\downarrow 3.15$ & 0.050 & $\downarrow 2.70$ & 0.11 & Energy-related function \\
\hline $\begin{array}{l}\text { Pyroglutamic acid or glutamine or } \\
\text { glutamate }\end{array}$ & 1.4 & RPLC & 130.0500 & {$[\mathrm{M}+\mathrm{H}]^{+}$} & 1 & 4 & 个 1.97 & 0.022 & $\uparrow 1.22$ & 0.29 & $\uparrow 2.40$ & 0.0002 & Glutathione metabolism \\
\hline S-formylglutathione & 1.1 & RPLC & 176.1031 & {$[\mathrm{M}+\mathrm{H}+\mathrm{Na}]^{2+}$} & 5 & 1 & $\uparrow 1.88$ & 0.15 & $\uparrow 1.26$ & 0.49 & 个 2.37 & 0.024 & Glutathione metabolism \\
\hline $\mathrm{PE}(\mathrm{O}-36: 6) / \mathrm{PE}(\mathrm{P}-36: 5)$ & 28.2 & RPLC & 722.5095 & {$[\mathrm{M}+\mathrm{H}]^{+}$} & 3 & 1 & 个 1.39 & 0.22 & 个 1.35 & 0.17 & 个 1.88 & 0.050 & Glycerophospholipid metabolism \\
\hline $\mathrm{PE}(0-38: 6) / \mathrm{PE}(\mathrm{P}-38: 5)$ & 28.2 & RPLC & 750.5419 & {$[\mathrm{M}+\mathrm{H}]^{+}$} & 1 & 1 & $\uparrow 1.48$ & 0.22 & $\uparrow 1.25$ & 0.72 & $\uparrow 1.85$ & 0.051 & Glycerophospholipid metabolism \\
\hline $\mathrm{PS}(\mathrm{P}-29: 0)$ & 6.2 & HILIC & 678.4701 & {$[\mathrm{M}+\mathrm{H}]^{+}$} & 0 & 1 & $\uparrow 1.59$ & 0.050 & $\downarrow 1.06$ & 0.75 & $\uparrow 1.50$ & 0.076 & Glycerophospholipid metabolism \\
\hline L-lactic acid & 1.8 & RPLC & 135.0029 & {$[\mathrm{M}+\mathrm{Na}]^{+}$} & 0 & 1 & $\uparrow 2.41$ & 0.026 & $\downarrow 1.76$ & 0.40 & $\uparrow 1.37$ & 0.17 & Glycolysis/Energy metabolism \\
\hline Beta-D-Fructose 1,6-bisphosphate & 0.9 & HILIC & 360.9726 & {$[\mathrm{M}+\mathrm{Na}-2 \mathrm{H}]^{-}$} & 5 & 1 & $\downarrow 7.36$ & 0.019 & 个 2.42 & 0.20 & $\downarrow 3.03$ & 0.10 & Glycolysis/Energy metabolism \\
\hline L-Tryptophan & 12.7 & RPLC & 205.0973 & {$[\mathrm{M}+\mathrm{H}]^{+}$} & 1 & 5 & 个 1.24 & 0.60 & 个 1.14 & 0.81 & $\uparrow 1.49$ & 0.051 & Serotonin precursor \\
\hline \multirow{2}{*}{ L-Glutamine } & 1.4 & RPLC & 147.0765 & {$[\mathrm{M}+\mathrm{H}]^{+}$} & 1 & \multirow[b]{2}{*}{5} & $\uparrow 2.01$ & 0.084 & $\uparrow 1.28$ & 0.33 & $\uparrow 2.58$ & 0.0003 & \multirow{2}{*}{ Glutamate precursor } \\
\hline & 1.35 & RPLC & 145.0612 & {$[\mathrm{M}-\mathrm{H}]^{-}$} & 4 & & $\uparrow 2.35$ & 0.12 & $\uparrow 1.25$ & 0.55 & $\uparrow 2.94$ & 0.0013 & \\
\hline L-Glutamic acid & 1.5 & RPLC & 148.0605 & {$[\mathrm{M}+\mathrm{H}]^{+}$} & 0 & 5 & $\uparrow 2.16$ & 0.040 & $\uparrow 1.67$ & 0.021 & 个 3.62 & $<0.0001$ & Neurotransmission \\
\hline $\mathrm{N}$-stearoyl GABA & 20.5 & RPLC & 370.3316 & {$[\mathrm{M}+\mathrm{H}]^{+}$} & 0 & 4 & $\downarrow 1.35$ & 0.28 & 个 1.50 & 0.052 & $\uparrow 1.11$ & 0.18 & Neurotransmission \\
\hline N-Acetyl-L-aspartate & 2.0 & RPLC & 176.0556 & {$[\mathrm{M}+\mathrm{H}]^{+}$} & 1 & 4 & $\uparrow 1.37$ & 0.023 & $\uparrow 1.63$ & 0.027 & $\uparrow 2.23$ & 0.0027 & Neurotransmission \\
\hline Tyramine & 9.7 & RPLC & 120.0810 & {$\left[\mathrm{M}+\mathrm{H}-\mathrm{H}_{2} \mathrm{O}\right]^{+}$} & 2 & 4 & $\downarrow 1.19$ & 0.75 & $\downarrow 1.42$ & 0.29 & $\downarrow 1.69$ & 0.041 & Neurotransmission \\
\hline GABA & 1.4 & RPLC & 102.0552 & {$[\mathrm{M}-\mathrm{H}]^{-}$} & 8 & 5 & $\uparrow 2.33$ & 0.068 & 个 2.22 & 0.0065 & 个 5.19 & $<0.0001$ & Neurotransmission \\
\hline 5-Deoxyadenosine & 2.2 & RPLC & 288.0516 & {$[\mathrm{M}+\mathrm{K}-2 \mathrm{H}]^{-}$} & 4 & 1 & $\uparrow 3.52$ & 0.068 & $\uparrow 1.40$ & 0.37 & 个 4.93 & 0.0003 & Purine degradation/salvage \\
\hline 5-Phosphoribosylamine & 2.17 & RPLC & 228.0301 & {$[\mathrm{M}-\mathrm{H}]^{-}$} & 9 & 1 & $\uparrow 3.83$ & 0.071 & $\uparrow 1.38$ & 0.41 & $\uparrow 5.30$ & 0.0004 & Purine synthesis \\
\hline \multirow{2}{*}{ Xanthine } & 1.6 & RPLC & 151.0254 & [M-H]- & 5 & 2 & 个 5.45 & 0.055 & $\uparrow 1.54$ & 0.41 & $\uparrow 8.42$ & 0.014 & Purine degradation \\
\hline & 2.14 & HILIC & 151.0254 & {$[\mathrm{M}-\mathrm{H}]^{-}$} & 4 & 2 & 个 8.76 & 0.078 & 个 1.96 & 0.28 & $\uparrow 17.14$ & 0.050 & Purine degradation \\
\hline 2'-Deoxyinosine 5'-phosphate (dIMP) & 2.1 & HILIC & 331.0453 & {$[\mathrm{M}-\mathrm{H}]^{-}$} & 1 & 1 & $\downarrow 1.31$ & 0.20 & $\downarrow 1.33$ & 0.18 & $\downarrow 1.74$ & 0.041 & Purine degradation/salvage \\
\hline 5,6-dihydrouridine & 1.4 & RPLC & 227.0650 & {$[\mathrm{M}-\mathrm{H} 2 \mathrm{O}-\mathrm{H}]^{-}$} & 7 & 1 & $\uparrow 2.10$ & 0.14 & 个 1.30 & 0.45 & $\uparrow 2.73$ & 0.0012 & Purine metabolism/tRNA synthesis \\
\hline
\end{tabular}




\begin{tabular}{|c|c|c|c|c|c|c|c|c|c|c|c|c|c|}
\hline \multirow{2}{*}{ Compound } & \multirow{2}{*}{ Rt (min) } & \multirow{2}{*}{ LC method } & \multirow{2}{*}{$\mathrm{m} / \mathrm{z}$} & \multirow{2}{*}{ Adduct } & \multirow{2}{*}{$\begin{array}{c}\text { Mass error } \\
{[p p m]}\end{array}$} & \multirow{2}{*}{ Level $\dagger$} & \multicolumn{2}{|c|}{ IV vs. PM T $T_{0}$} & \multicolumn{2}{|c|}{$\mathrm{PM} \mathrm{T}_{0}$ vs. $\mathrm{T}_{30}$} & \multicolumn{2}{|c|}{ IV vs. PM T $T_{30}$} & \multirow{2}{*}{ Pathway/Function } \\
\hline & & & & & & & FC & $p$-value & FC & $p$-value & FC & $p$-value & \\
\hline 3-Deoxyvitamin D3 & 25.4 & RPLC & 369.3516 & {$[\mathrm{M}+\mathrm{H}]^{+}$} & 0 & 4 & $\uparrow 1.37$ & 0.084 & $\uparrow 1.03$ & 0.90 & $\uparrow 1.45$ & 0.050 & Vitamin D metabolism \\
\hline 1,25-dihydroxy-22-oxavitamin D3 & 25.8 & RPLC & 419.3156 & {$[\mathrm{M}+\mathrm{H}]^{+}$} & 0 & 1 & $\uparrow 1.16$ & 0.65 & $\uparrow 1.27$ & 0.010 & 个 1.47 & 0.22 & Vitamin D metabolism \\
\hline $1,11,25$-trihydroxyvitamin D3 & 26.2 & RPLC & 433.3313 & {$[\mathrm{M}+\mathrm{H}]^{+}$} & 0 & 1 & $\uparrow 1.12$ & 0.73 & $\uparrow 1.61$ & 0.011 & $\uparrow 1.79$ & 0.068 & Vitamin D metabolism \\
\hline N-Formyl-L-glutamate & 1.4 & RPLC & 176.0555 & {$[\mathrm{M}+\mathrm{H}]^{+}$} & 1 & 1 & $\downarrow 1.75$ & 0.0069 & $\uparrow 1.78$ & 0.025 & $\uparrow 1.02$ & 0.90 & Histidine metabolism \\
\hline Dihydroxy-3-methylvalerate & 0.9 & HILIC & 129.0548 & {$[\mathrm{M}-\mathrm{H} 2 \mathrm{O}-\mathrm{H}]^{-}$} & 2 & 1 & $\downarrow 3.77$ & 0.0033 & $\uparrow 1.07$ & 0.62 & $\downarrow 3.51$ & 0.010 & BCAA metabolism \\
\hline L-Leucine/Isoleucine & 7.7 & RPLC & 132.1020 & {$[\mathrm{M}+\mathrm{H}]^{+}$} & 1 & 5 & $\uparrow 1.02$ & 0.95 & $\uparrow 1.40$ & 0.36 & $\uparrow 1.43$ & 0.055 & BCAA metabolism \\
\hline L-Phenylalanine & 9.7 & RPLC & 166.0864 & {$[\mathrm{M}+\mathrm{H}]^{+}$} & 1 & 5 & $\downarrow 1.28$ & 0.33 & $\downarrow 1.31$ & 0.23 & $\downarrow 1.73$ & 0.050 & AAA metabolism \\
\hline L-Tyrosine & 7.6 & RPLC & 182.0813 & {$[\mathrm{M}+\mathrm{H}]^{+}$} & 1 & 5 & $\downarrow 1.11$ & 0.78 & $\downarrow 1.55$ & 0.045 & $\downarrow 1.68$ & 0.047 & AAA metabolism \\
\hline LacCer(d30:0) & 28.2 & RPLC & 790.5646 & {$[\mathrm{M}+\mathrm{H}-\mathrm{H} 2 \mathrm{O}]^{+}$} & 2 & 1 & $\uparrow 1.36$ & 0.31 & $\uparrow 1.33$ & 0.47 & $\uparrow 1.81$ & 0.040 & $\begin{array}{l}\text { Regulation of permeability of } \\
\text { blood-brain barrier }\end{array}$ \\
\hline $\mathrm{PA}(0-38: 6) / \mathrm{PA}(\mathrm{P}-38: 5)$ & 28.2 & RPLC & 707.4996 & {$[\mathrm{M}+\mathrm{H}]^{+}$} & 2 & 1 & $\uparrow 1.36$ & 0.42 & $\uparrow 1.30$ & 0.64 & $\uparrow 1.77$ & 0.044 & Mediators in brain functions \\
\hline $\mathrm{PA}(45: 8) / \mathrm{PA}(0-45: 9)$ & 28.2 & RPLC & 815.5606 & {$[\mathrm{M}+\mathrm{H}]^{+}$} & 1 & 1 & $\uparrow 1.28$ & 0.69 & $\uparrow 1.67$ & 0.25 & $\uparrow 2.12$ & 0.050 & Mediators in brain functions \\
\hline MGDG(36:5) & 28.2 & RPLC & 777.5521 & {$[\mathrm{M}+\mathrm{H}]^{+}$} & 1 & 1 & $\uparrow 1.73$ & 0.12 & 个 1.31 & 0.26 & $\uparrow 2.26$ & 0.043 & Myelin component \\
\hline Betaine & 1.9 & RPLC & 156.0422 & {$[\mathrm{M}+\mathrm{K}]^{+}$} & 0 & 4 & $\uparrow 1.36$ & 0.56 & $\uparrow 1.12$ & 0.91 & $\uparrow 1.50$ & 0.049 & Osmolytic activity \\
\hline 4,5-dehydrodocosahexaenoic acid & 1.1 & HILIC & 344.2616 & {$[\mathrm{M}+\mathrm{H}-\mathrm{H} 2 \mathrm{O}]^{+}$} & 1 & 1 & $\uparrow 2.18$ & 0.24 & $\uparrow 4.29$ & 0.058 & $\uparrow 9.33$ & 0.053 & Docosahexaenoic acid metabolism \\
\hline Acetyl-L-carnitine & 9.4 & RPLC & 204.1232 & {$[\mathrm{M}+\mathrm{H}]^{+}$} & 1 & 4 & $\uparrow 1.56$ & 0.30 & $\uparrow 1.21$ & 0.53 & 个 1.89 & 0.0087 & Acetyl-CoA transport \\
\hline $\begin{array}{l}\text { Deoxyribose 1-phosphate/Deoxyribose } \\
\text { 5-monophosphate }\end{array}$ & 2.1 & HILIC & 427.0448 & {$[2 \mathrm{M}-\mathrm{H}]^{-}$} & 8 & 1 & $\downarrow 1.65$ & 0.023 & $\downarrow 1.22$ & 0.43 & $\downarrow 2.01$ & 0.022 & $\begin{array}{l}\text { Pyrimidine metabolism/pentose } \\
\text { phosphate pathway }\end{array}$ \\
\hline L-Citrulline & 1.5 & RPLC & 176.1031 & {$[\mathrm{M}+\mathrm{H}]^{+}$} & 1 & 5 & $\downarrow 1.36$ & 0.24 & $\downarrow 1.17$ & 0.54 & $\downarrow 1.44$ & 0.051 & Urea cycle/NO generation \\
\hline
\end{tabular}

\section{; BCAA - branched-chain}

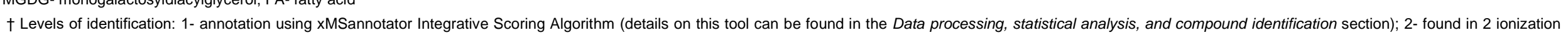
modes, 2 employed chromatography types or combination of those; 3 - found in 3 methods (e.g. two ionization polarities in RPLC and 1 polarity in HILIC); 4 - MS/MS spectrum match with database or Rt match with standard; 5 -

FC- fold change; $p$-values represent false discovery rate adjusted values 


\section{Boxplots for the discussed metabolites}
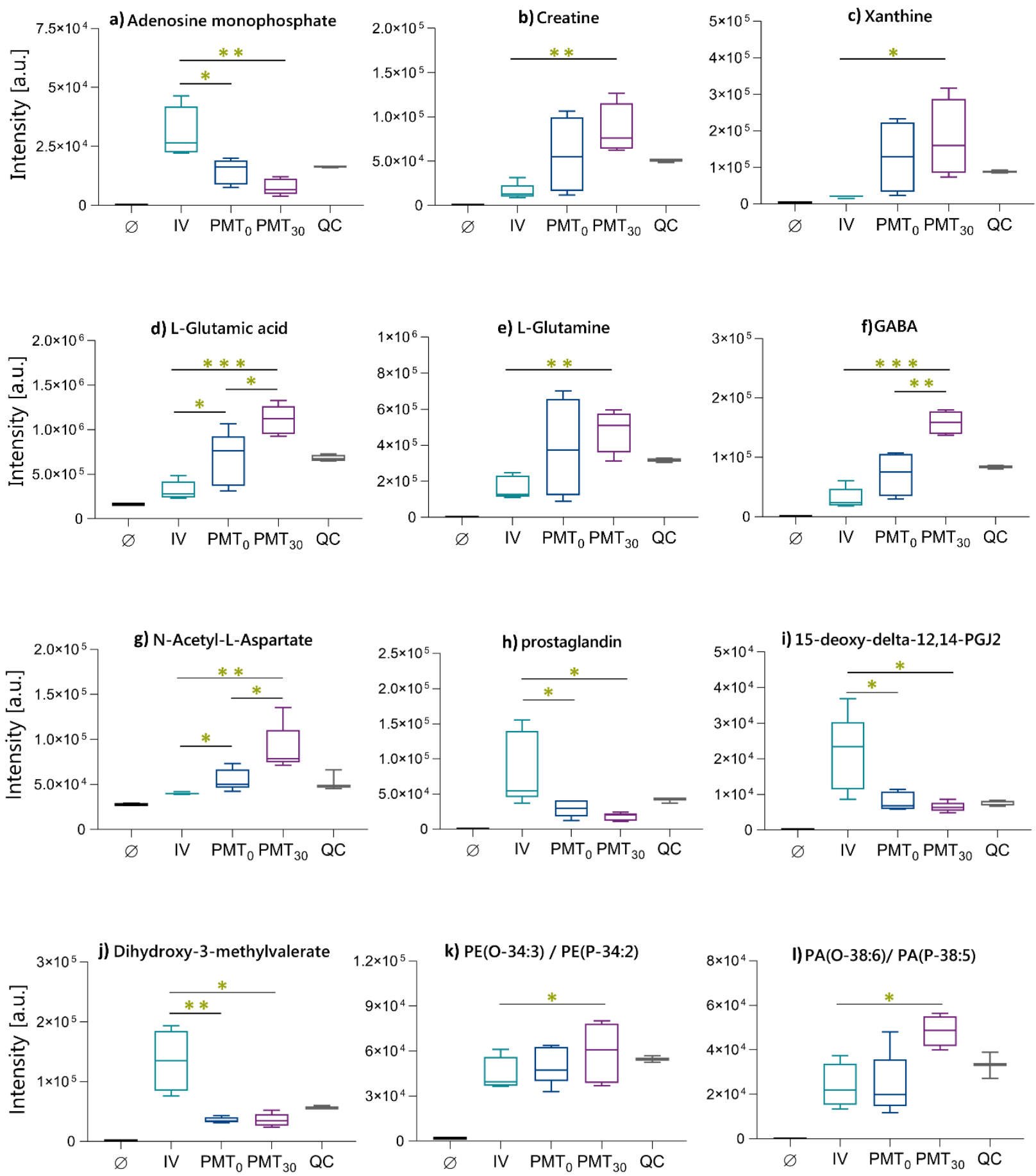

Figure S5. Boxplots of key metabolites discussed in the main text (shown as absolute intensities) and how their distribution varies across the sampling timepoints. Q - SPME fiber blank; IV - in vivo sampling; PMT 0 -postmortem sampling at 0 min of PMI; $\mathrm{PMT}_{30}$-postmortem sampling at 30 min of PMI; QC - pooled quality control samples; ${ }^{\star}-p<0.05 ;{ }^{* \star}-p<0.005 ;{ }^{* \star *} p<0.0001$. 


\section{Confirmation of metabolites' identities by MS/MS - comparison with real standards}
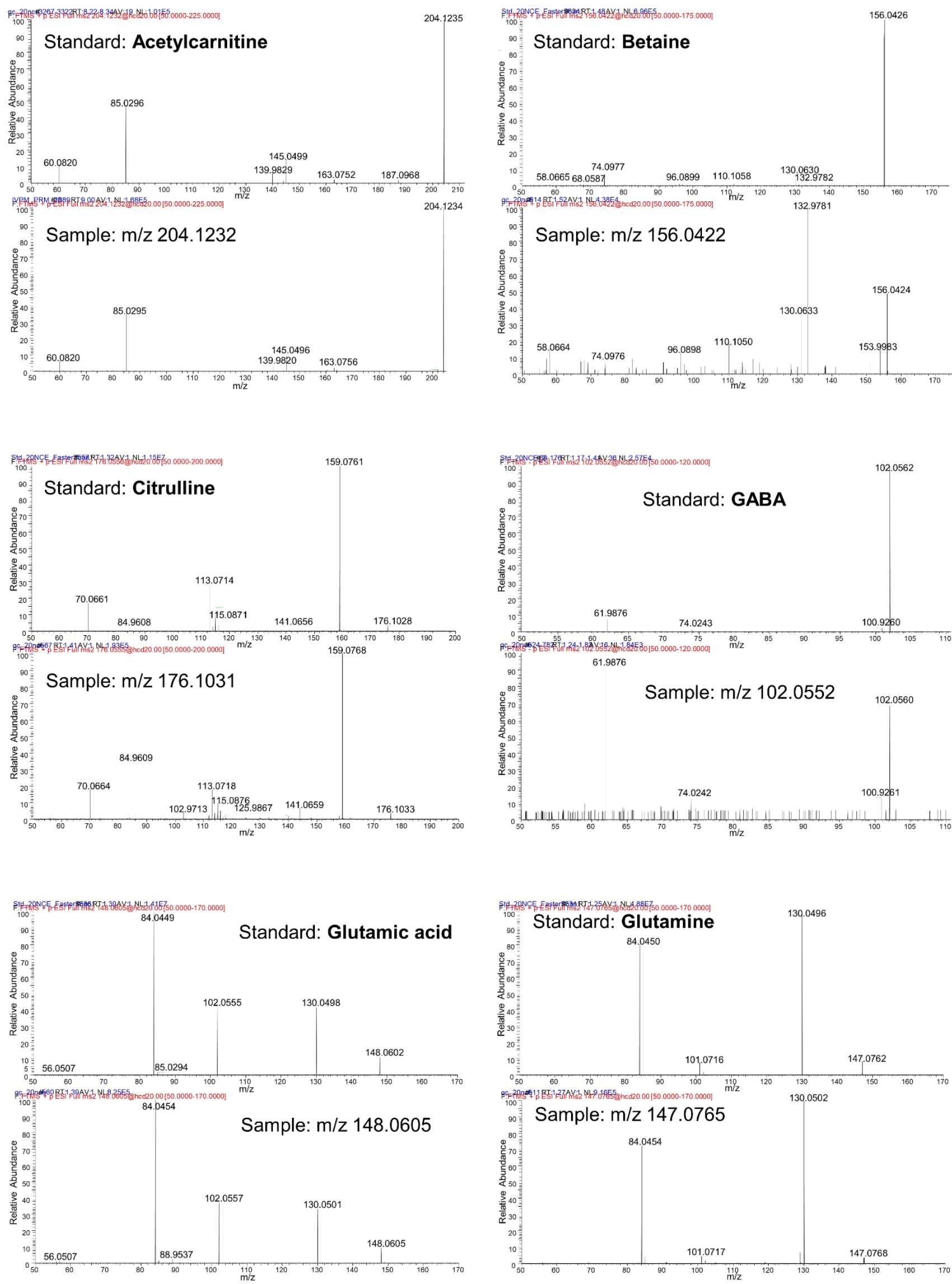

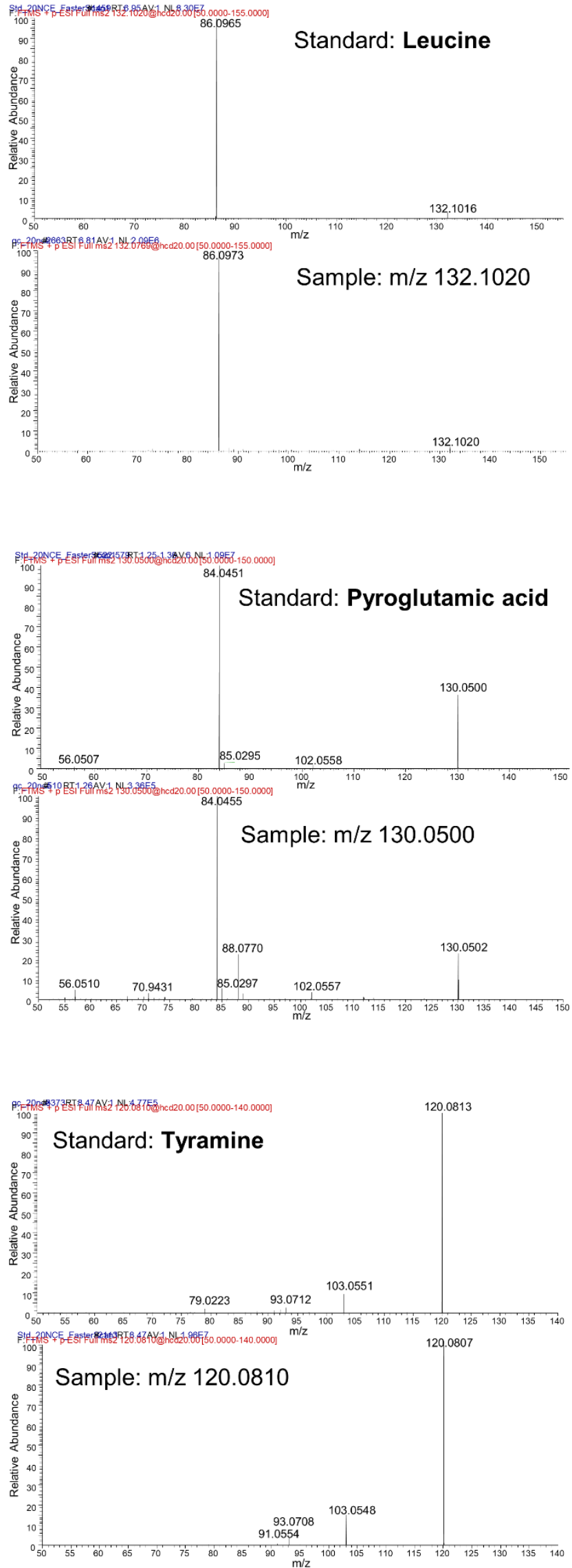
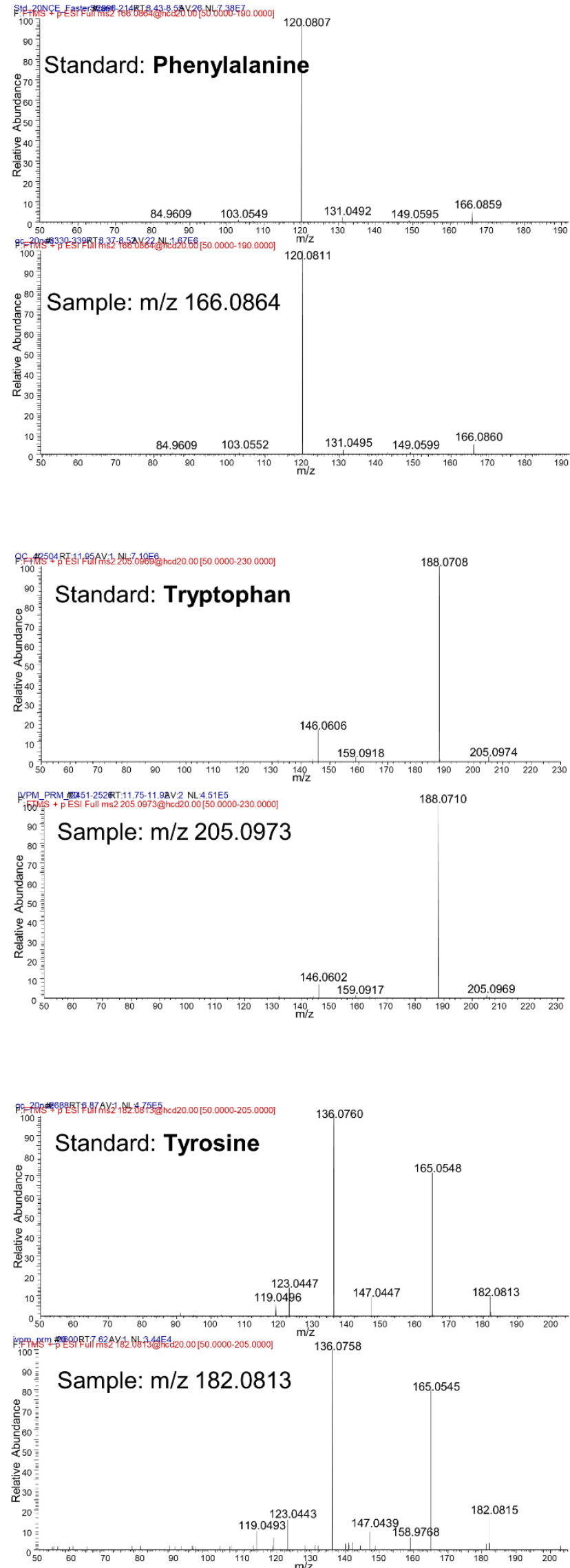


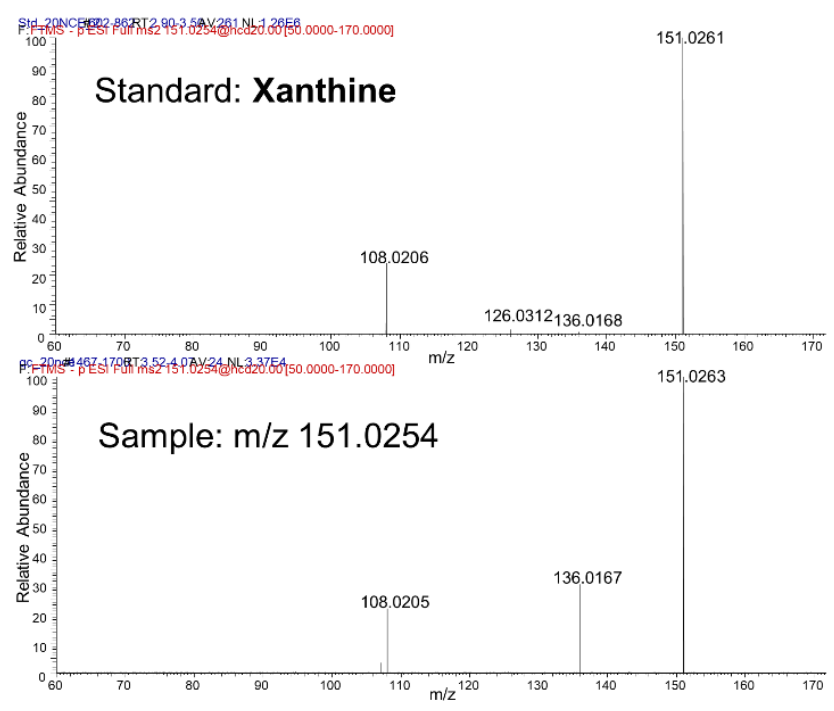

Confirmation of metabolites' identities by MS/MS - comparison with databases
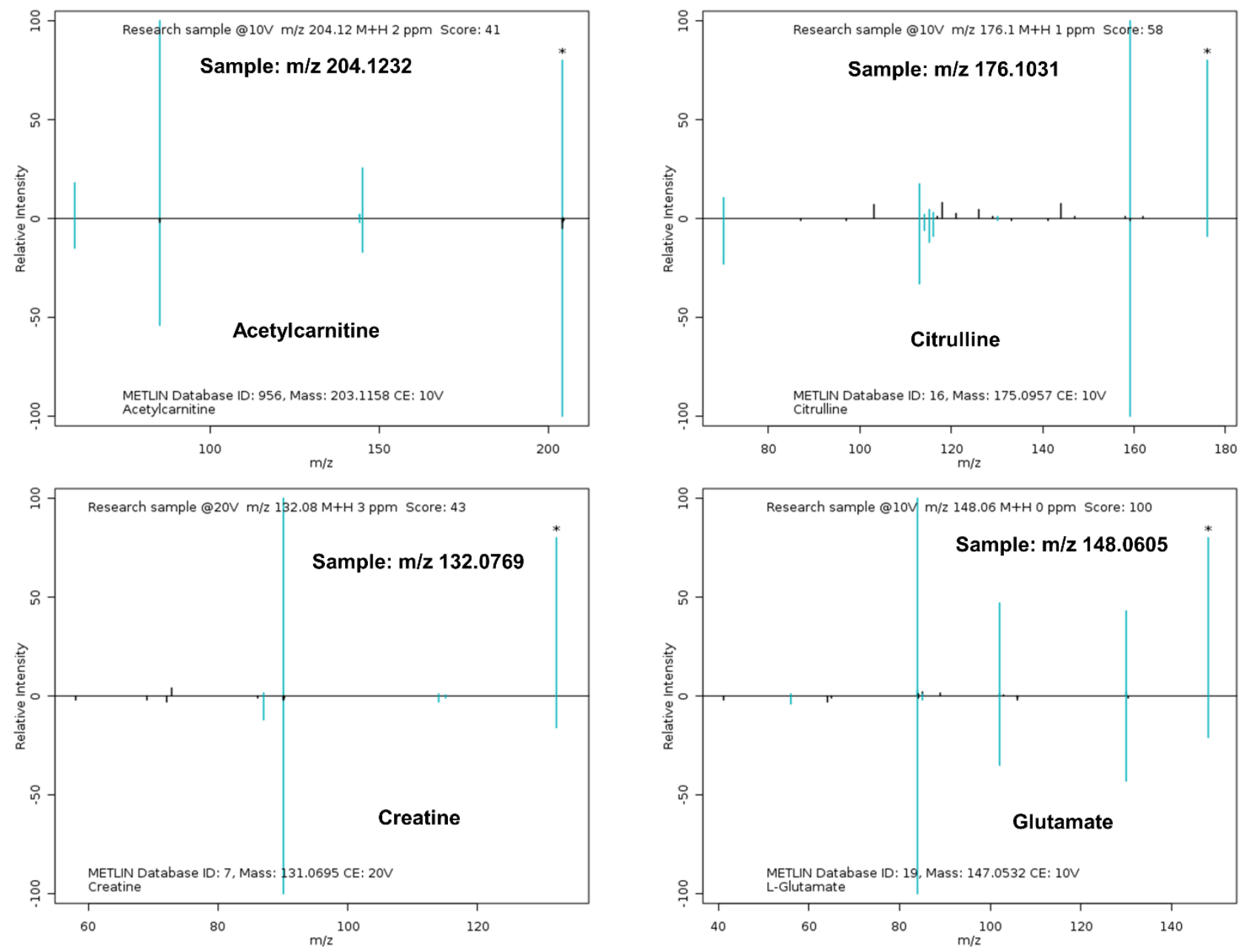

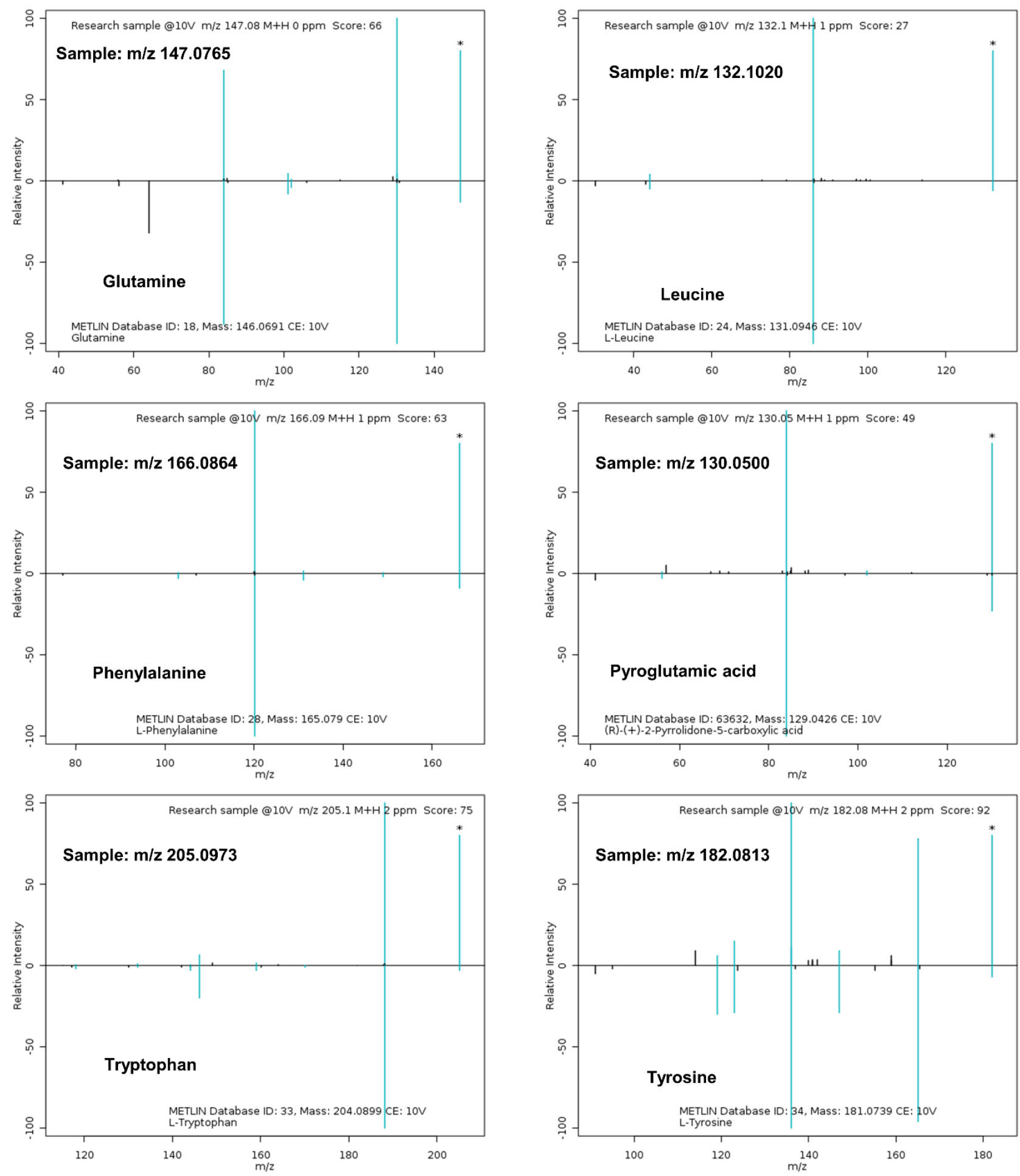

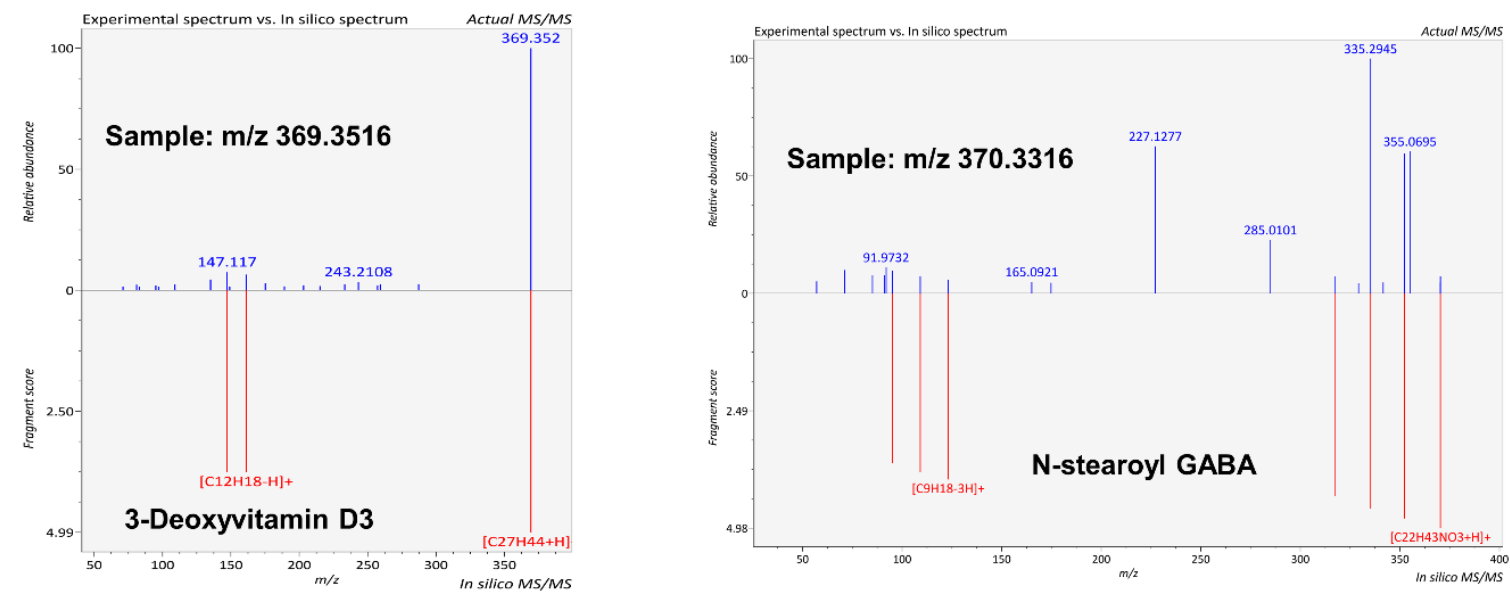

Table S4: MS/MS fragments related to metabolites which could not be confirmed by comparison with real standards or database search.

\begin{tabular}{|c|c|c|c|c|c|}
\hline Compound & $\begin{array}{c}\text { Rt } \\
{[\mathrm{min}]}\end{array}$ & $\mathrm{m} / \mathrm{z}$ & adduct & $\begin{array}{l}\text { Mass error } \\
\text { [ppm] }\end{array}$ & Fragments $\mathrm{m} / \mathrm{z}$ \\
\hline GlcCer(d40:1)/GalCer(d40:1) & 26.1 & 784.6661 & {$[\mathrm{M}+\mathrm{H}]+$} & 0 & $483.3689,511.3974$ \\
\hline FA 17:4 & 18.4 & 263.2006 & {$[\mathrm{M}+\mathrm{H}-\mathrm{H} 2 \mathrm{O}]+$} & 1 & $57.0346,91.9731,207.1382,245.2267$ \\
\hline 1,25-dihydroxy-22-oxavitamin D3 & 25.8 & 419.3156 & {$[\mathrm{M}+\mathrm{H}]+$} & 0 & $\begin{array}{c}\text { 71.0863, } 85.1019,127.1484,149.0235 \\
167.0340,293.1741\end{array}$ \\
\hline $\mathrm{PA}(0-38: 6) / \mathrm{PA}(\mathrm{P}-38: 5)$ & 28.2 & 815.5606 & {$[\mathrm{M}+2 \mathrm{Na}-\mathrm{H}]+$} & 4 & $\begin{array}{c}167.0560,239.0947,299.0622,355.0699 \\
415.0374,503.1109\end{array}$ \\
\hline N-Acetyl-L-aspartate & 2.0 & 176.0556 & {$[\mathrm{M}+\mathrm{H}]+$} & 1 & $\begin{array}{c}74.0248,88.0403,116.0349,130.0499 \\
134.0449,158.0447\end{array}$ \\
\hline 15-deoxy-delta-12,14-Prostaglandin J2 & 15.2 & 317.2115 & {$[\mathrm{M}+\mathrm{H}]+$} & 1 & $\begin{array}{c}111.0448,129.0552,171.1384,281.1755 \\
299.1850\end{array}$ \\
\hline 1,11,25-trihydroxyvitamin D3 & 26.2 & 433.3313 & {$[\mathrm{M}+\mathrm{H}]+$} & 0 & $\begin{array}{c}\text { 71.0866, } 5.1024,98.9852,119.0864,210.9508 \\
369.2159,380.7238\end{array}$ \\
\hline $\mathrm{PE}(\mathrm{O}-38: 6) / \mathrm{PE}(\mathrm{P}-38: 5)$ & 28.2 & 750.5419 & {$[\mathrm{M}+\mathrm{H}]+$} & 1 & $88.0769,551.3280,607.3917,663.4521$ \\
\hline 5,6-dihydrouridine & 1.4 & 227.0650 & {$[\mathrm{M}-\mathrm{H} 2 \mathrm{O}-\mathrm{H}]-$} & 7 & $127.0503,145.0610,166.9915$ \\
\hline 5-phosphoribosylamine & 2.2 & 228.0301 & {$[\mathrm{M}-\mathrm{H}]-$} & 9 & $88.0392,112.0505,130.0613,174.9919$ \\
\hline 15-deoxy-delta-12,14-Prostaglandin J2 & 15.2 & 315.1969 & {$[\mathrm{M}-\mathrm{H}]-$} & 0 & $59.0124,116.9275,232.9251,254.9072$ \\
\hline Dihydroxy-3-methylvalerate & 0.9 & 129.0548 & [M-H-H2O]- & 0 & $57.0337,83.0500,111.0454$ \\
\hline Prostaglandin F $2 \alpha$ & 0.8 & 353.2333 & {$[\mathrm{M}-\mathrm{H}]-$} & 2 & $96.9763,116.9288,146.9831,255.2333$ \\
\hline
\end{tabular}

\section{References}

(1) Craig, A.; Cloarec, O.; Holmes, E.; Nicholson, J. K.; Lindon, J. C. Scaling and Normalization Effects in NMR Spectroscopic Metabonomic Data Sets. Anal. Chem. 2006, 78 (7), 2262-2267.

(2) Berg, R. A. Van Den; Hoefsloot, H. C. J.; Westerhuis, J. A.; Smilde, A. K.; Werf, M. J. Van Der. Centering , Scaling and Transformations : Improving the Biological Information Content of Metabolomics Data. BMC Genomics 2006, 7 (142), 1-15.

(3) Chong, J.; Wishart, D. S.; Xia, J. Using MetaboAnalyst 4.0 for Comprehensive and Integrative Metabolomics Data Analysis. Curr. Protoc. Bioinforma. 2019, 68, 1-128. 